\title{
An Impedance-based Algorithm to Detect Ventilations During Cardiopulmonary Resuscitation
}

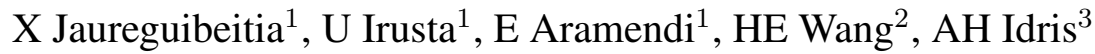 \\ ${ }^{1}$ University of the Basque Country (UPV/EHU), Bilbao, Spain \\ ${ }^{2}$ University of Texas Health Science Center, Houston (Texas), USA \\ ${ }^{3}$ University of Texas Southwestern Medical Center, Dallas (Texas), USA
}

\begin{abstract}
Cardiopulmonary resuscitation $(C P R)$ is a core therapy to treat out-of-hospital cardiac arrest (OHCA). Thoracic impedance (TI) can be used to assess ventilations during CPR, but the signal is also affected by chest compression (CC) artifacts. This study presents a method for TI-based ventilation detection during concurrent manual CCs.

Data from 152 OHCA patients were analyzed. A total of 423 TI segments of at least $60 \mathrm{~s}$ during ongoing CCs were extracted. True ventilations were annotated using the capnogram. The final dataset comprised $1210 \mathrm{~min}$ of TI recordings and 9665 ground truth ventilations.

A three-stage detection algorithm was developed. First, the TI signal was filtered to obtain ventilation waveforms, including a least mean squares filter to remove artifacts due to CCs. Potential ventilations were then identified with a heuristic detector and characterized by a set of 16 features. These were finally fed to a random forest classifier to discriminate between true ventilations and false positives.

Patients were split into 100 distinct training (70\%) and test (30\%) partitions. The median (interquartile range) sensitivity, PPV and F-score were 83.9 (70.2-91.2)\%, 86.1 (75.0-93.3)\% and 84.3 (72.1-91.4)\%. Our method would allow feedback on ventilation rates as well as surrogate measures of insufflated air volume during CPR.
\end{abstract}

\section{Introduction}

High-quality cardiopulmonary resuscitation (CPR) is a key element in the treatment of out-of-hospital cardiac arrest (OHCA). Chest compressions (CC) and ventilations induce an artificial flow of oxygenated blood in the patient which delays the progress of ischemia. This buys time for other therapies to be applied and greatly improves the odds of survival [1]. Resuscitation guidelines recommend CCs to be delivered at rates of $100-120 \mathrm{~min}^{-1}$ [1]. When CCs are performed without interruption, ventilations should be delivered at a rate of about $10 \mathrm{~min}^{-1}$, avoiding hyperventi- lation [2]. Adherence to these recommendations improves when rescuers are provided with real-time feedback on CPR therapy [3]. Many solutions have been implemented to enhance CC quality, from integrated accelerometers to monitor rates and depths, to automated devices that compress the chest mechanically. In contrast, few solutions exist to provide feedback on ventilation quality.

Capnography, which monitors the concentration of $\mathrm{CO}_{2}$ in expired gases, is the recommended method to assess ventilations, but requires advanced airway management and is therefore rarely available early during resuscitation. Thoracic impedance (TI), a measure of body resistance to current flow, fluctuates with air volume changes in the lungs and can also be used to monitor ventilations. TI is typically available much earlier, as it is recorded by most defibrillation equipment concurrently with the ECG. Moreover, the amplitudes of the ventilation waveforms in the TI can be taken as surrogate measures of insufflated air volumes and can be associated with survival outcomes [4].

Detecting ventilations in the TI is challenging even in intervals free of CCs [5]. TI fluctuations vary significantly between cases, and the signal itself is sensitive to noise sources like patient or cable movements. Moreover, CCs produce large artifacts that have to be removed to detect ventilations. State-of-the-art solutions for this scenario make use of adaptive filters to suppress CC artifacts [6]. The present work was built on a previous machine learning solution for ventilation detection during mechanical CCs [7]. The challenge was in adapting those methods to manual CPR, in which CC patterns are much more variable due to rescuer and patient diversity.

\section{Data materials}

The study dataset included data from 152 OHCA cases treated by the emergency medical services of the Dallas Fort Worth area and enrolled in the Pragmatic Airway Resuscitation Trial [8]. All cases were treated and recorded with a Philips MRx monitor-defibrillator (Philips Medical Systems, Andover, MA), which acquires the TI signal with 
a $200 \mathrm{~Hz}$ sampling frequency and a resolution of $0.74 \mathrm{~m} \Omega$. The electronic recordings were converted to a MATLAB (MathWorks Inc., Natick, MA) format, and explored to locate TI segments suitable for the study design. Segments of at least $60 \mathrm{~s}$ with ongoing $\mathrm{CCs}$ were considered. Intervals presenting abrupt TI excursions or CC pauses longer than $20 \mathrm{~s}$ were discarded. Only segments with synchronous recordings of compression depth (CD) and capnography were included. CC instants were annotated in the CD and used as reference to filter out the $\mathrm{CC}$ artifact. Ventilations were annotated in the capnogram and set as ground truth to develop the detection algorithm. Time delays between the capnogram and the TI were corrected. Figure 1 shows an example of the signals involved in the annotation process. A total of 423 TI segments were extracted, comprising $1210 \mathrm{~min}$ of signal recordings and 9665 ground truth ventilations (97.7\% during $\mathrm{CCs}$ ).

\section{Methods}

A three-stage method was designed to detect ventilations. First, the TI was preprocessed to filter out CC artifacts. A peak detection procedure was then applied to identify potential ventilations in the resulting $s_{\mathrm{v}}(n)$ signal (see Fig 1c). The waveform fluctuation around each peak was delimited and characterized by a set of 16 features. Finally, a random forest classifier was used to discriminate between true ventilations and the many false positives output by the previous stage.

\subsection{Signal preprocessing}

The impedance signal was first downsampled to $50 \mathrm{~Hz}$. A $0.06 \mathrm{~Hz}$ cutoff high-pass IIR filter was then applied to remove the DC offset, followed by a moving average filter ( $L=50)$ to remove high-frequency components, including most of the $\mathrm{CC}$ artifact. The resulting signal $s(n)$ is assumed to be given by $s(n)=s_{\mathrm{v}}(n)+s_{\mathrm{cc}}(n)$, where $s_{\mathrm{v}}(n)$ contains the desired ventilation information and $s_{\mathrm{cc}}(n)$ represents the residual of the $\mathrm{CC}$ artifact. A Least Mean Squares (LMS) adaptive filter, following the model proposed by Irusta et al [9], was applied to remove $\mathrm{CC}$ artifacts. The CC component was approximated by a quasi-periodic signal of the form:

$\hat{s}_{\mathrm{cc}}(n)=A(n) \sum_{k=1}^{N}\left[a_{k}(n) \cos (k \phi(n))+b_{k}(n) \sin (k \phi(n))\right]$

where $N$ is the number of harmonics. The phase term $\phi(n)$ is built by producing a $2 \pi$ phase shift between each pair of consecutive compressions. $A(n)$ differentiates intervals with $(A=1)$ and without $(A=0) \mathrm{CCs}$, with a pause in compressions being defined as an interval longer than $1.5 \mathrm{~s}$ in absence of CCs. The time varying in-phase and quadrature amplitude coefficients, $a_{k}$ and $b_{k}$, were then estimated using the LMS algorithm for harmonic interference suppression [10]. In this setting, the error signal $e(n)=s(n)-\hat{s}_{\mathrm{cc}}(n)$ is an estimate of the desired $s_{\mathrm{v}}(n)$ component. Values of $N=3$ and step-size $\mu=0.05$ were selected according to some initial experiments.

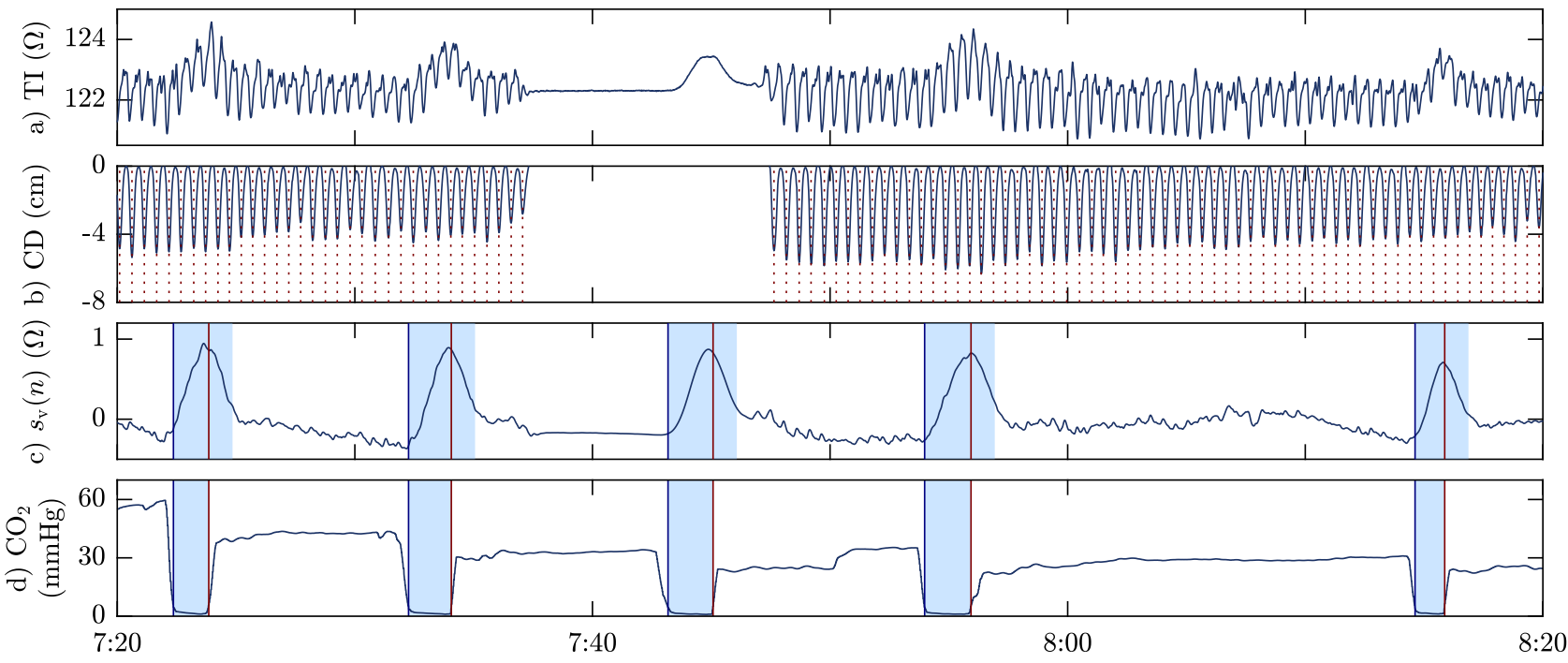

Figure 1: An example of the signals involved in the detection process. (a) The raw TI signal, as stored in defibrillator data. (b) The CD signal, including the annotated CC instants as dotted vertical lines. (c) The ventilation component of the TI signal, obtained through adaptive filtering using the CC instants shown in (b). (d) The time-aligned capnogram signal, including true ventilation annotations as shaded intervals. These intervals, associated to air insufflation phases, are reproduced in (c) and extended by up to $1 \mathrm{~s}$ to determine which peaks correspond to true ventilations. 


\subsection{Ventilation waveform processing}

Potential ventilations were detected as the highest local maxima of $s_{\mathrm{v}}(n)$ with a refractory period of $1.5 \mathrm{~s}$ (ventilation rate of $\left.40 \mathrm{~min}^{-1}\right)$. For each detected peak position $t_{p_{i}}$, the start and end points of the ventilation waveform, $t_{s_{i}}$ and $t_{e_{i}}$, were then estimated. The location of $t_{s_{i}}$ was first set to the minimum $s_{\mathrm{v}}(n)$ point in the interval $\left(t_{p_{i}}-5.5, t_{p_{i}}-0.45\right)$, and then adjusted following a series of heuristic rules. Amplitude relations between local maxima and minima within $\left(t_{s_{i}}, t_{p_{i}}\right)$ were evaluated to avoid merging several waveforms into a single detection. An initial slope condition was also applied to prevent $t_{s_{i}}$ from being set on a near flat signal region, far away from the actual waveform. An analogous procedure was followed to locate $t_{e_{i}}$ within the interval $\left(t_{p_{i}}+0.45, t_{p_{i}}+5.5\right)$. Any peak $t_{p_{i}}$ for which $t_{s_{i}}$ or $t_{e_{i}}$ could not be determined was discarded as potential ventilation.

\subsection{Feature extraction}

A total of 16 features were used to characterize each potential ventilation waveform. The first four corresponded to the amplitude and duration of the insufflation and exhalation phases, that is the intervals $\left(t_{s_{i}}, t_{p_{i}}\right)$ and $\left(t_{p_{i}}, t_{e_{i}}\right)$, respectively [7]. The next 10 features, 5 for each phase, were given by the coefficients of a least-squares approximation of the phase waveform in terms of up to fourth order Legendre polynomials. Curve fitting was approached assuming the samples of each phase to be equispaced over a $[-1,1]$ transformed time domain. Finally, the signal excursions $\Delta_{90}=P_{95}\left(s_{\mathrm{v}}\right)-P_{5}\left(s_{\mathrm{v}}\right)$ and $\Delta_{95}=P_{97.5}\left(s_{\mathrm{v}}\right)-$ $P_{2.5}\left(s_{\mathrm{v}}\right)$, which were computed in an up to $1 \mathrm{~min}$ interval around the peak position. $P_{\mathrm{x}}$ represents the $\mathrm{x}$-th amplitude percentile. This was deemed possible as windows of about $1 \mathrm{~min}$ are typically analyzed to report ventilation rates, and allowed the classifier to adapt to the large ventilation amplitude differences between cases.

\subsection{Classification}

The waveform detection stage was conceived to miss few true ventilations, so it produced a large number of false positives. A random forest classifier [11] was employed to discriminate between both cases. In order to train the classifier and test the algorithm, each potential ventilation $i$ reported by the detection stage was labeled as either true ventilation $\left(y_{i}=1\right)$ or false positive $\left(y_{i}=0\right)$ according to the ground truth annotations in the capnogram. Those waveforms with peak position $t_{p_{i}}$ within a ground truth interval (shaded regions in Figure 1c) were labeled as $y_{i}=1$. When several detections were reported within the same interval, only the one closest to the expiration onset (red lines in Figure 1c) was labeled as true ventilation. The out- puts of the detection stage formed a set of instance-label pairs $\left\{\boldsymbol{x}_{\boldsymbol{i}}, y_{i}\right\} \in \mathbb{R}^{K \times(0,1)}$, where $K=16$ is the number of features considered. A 100 tree ensemble size and a minimum leaf-size of 5 were used as classifier hyperparameters. Data was patient-wise weighted to avoid biasing results towards longer cases with a larger amount of ventilations.

\subsection{Model evaluation}

Data were randomly partitioned patient-wise into train $(70 \%)$ and test $(30 \%)$ sets. Due to large differences in the number of ventilations, the assignment was carried out in a quasi-stratified way, forcing the train set to contain between $65 \%$ and $75 \%$ of the ground truth ventilations. Performance was assessed in terms of sensitivity (Se, proportion of correctly identified true ventilations), positive predictive value (PPV, proportion of true ventilations among reported detections) and F-score $\left(\mathrm{F}_{1}\right.$, harmonic mean of Se and PPV). To avoid data partition bias in the results, 100 different train/test splits were generated and performance metrics were averaged patient-wise.

\section{Results}

The waveform detector overestimated ventilations with a median (interquartile range, IQR) patient-wise Se of $94.1(89.0-98.4) \%$ and a PPV of $56.0(44.9-66.7) \%$. The classification stage corrected most of the false positives, resulting in Se, PPV and $\mathrm{F}_{1}$ scores of 83.9 (70.291.2) \%, 86.1 (75.0-93.3) \% and 84.3 (72.1-91.4) \% respectively. The patient-wise distribution of performance metrics is shown in Figure 2.

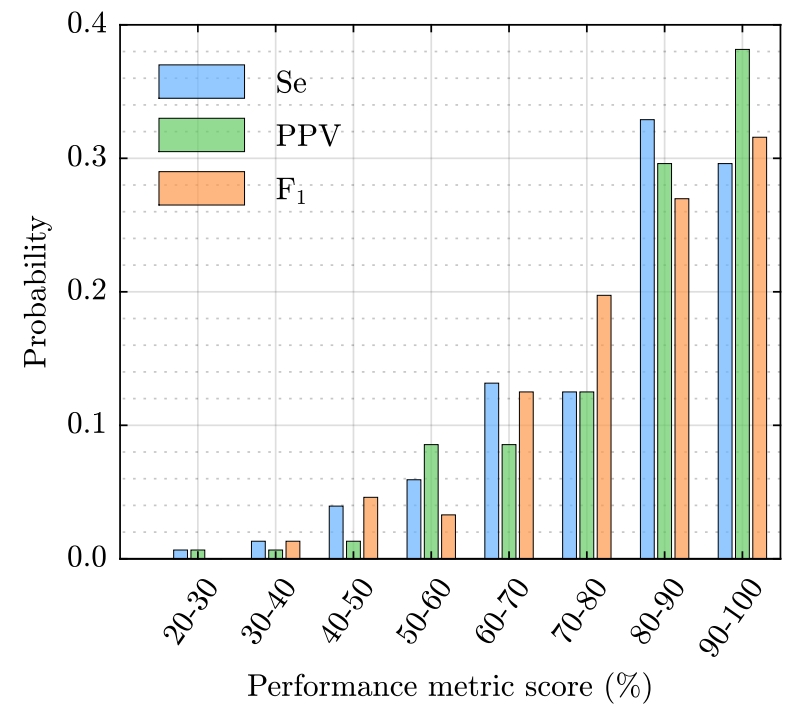

Figure 2: Patient-wise performance metric scores for the full algorithm, as probability distribution. 
Motion artifacts caused by rescuers during treatment were identified as the major error source. As shown in Figure 3, these may produce many false positives, and distort or even completely mask the actual ventilations.

\section{Conclusions}

An algorithm for impedance-based ventilation detection during concurrent manual CCs was demonstrated. With median patient-wise performance metric scores of about $85 \%$, the method could allow accurate feedback on ventilation rates and possibly on insufflated air volumes in the majority of cases. Performances could be improved in the future by employing more complex filters and feature selection techniques. For some cases, however, the algorithm presented a severely degraded performance due to motion artifacts, a phenomena not present during mechanical CPR [7], presumably due to the compression device being tightly attached to the patient. Devising a signal quality index to identify these situations may improve performance in the future. This would allow to anticipate problematic cases, avoiding inaccurate feedback, but more importantly, preventing the classifier from being fed with corrupted training examples which may severely hinder the overall performance.
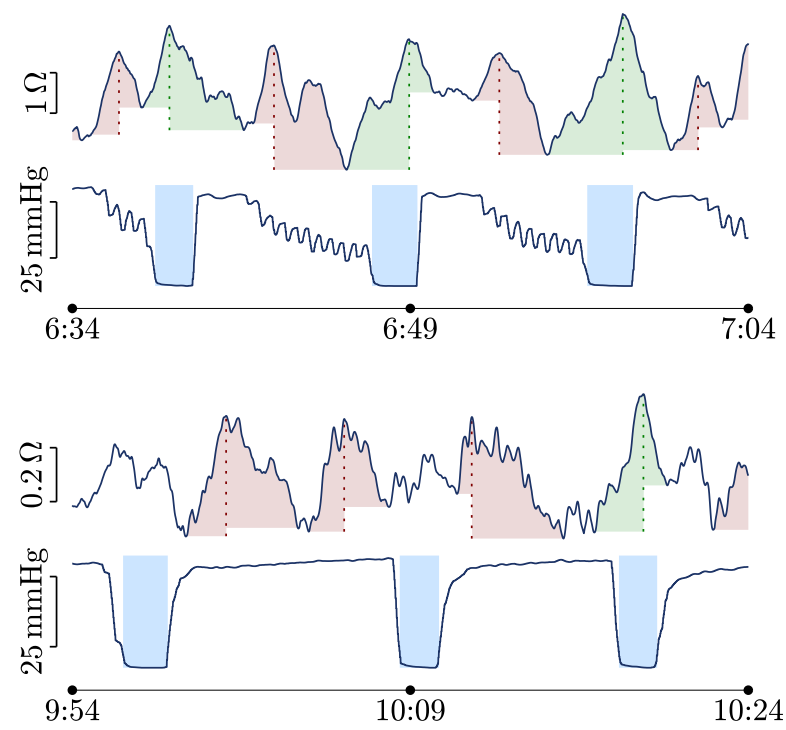

Figure 3: Examples of TI segments corrupted by motion artifacts. The detections output by the algorithm are displayed in in green for true ventilations and in red for false positives. For large ventilations (top), artifacts may result in additional detections and distorted waveforms. During shallow ventilations (bottom), artifacts might be dominant and result in extremely degraded performances.

\section{Acknowledgments}

This work was supported by the Spanish Ministerio de Ciencia, Innovacion y Universidades through grant RTI2018-101475-BI00, jointly with the Fondo Europeo de Desarrollo Regional (FEDER), and by the Basque Government through grants IT1229-19 and PRE-2019-1-0209.

\section{References}

[1] G. D. Perkins, A. J. Handley, R. W. Koster et al., "European resuscitation council guidelines for resuscitation 2015: Section 2. adult basic life support and automated external defibrillation." Resuscitation, vol. 95, pp. 81-99, Oct. 2015.

[2] J. Soar, J. P. Nolan, B. W. Böttiger et al., "European resuscitation council guidelines for resuscitation 2015: Section 3. adult advanced life support." Resuscitation, vol. 95, pp. 100-147, Oct. 2015.

[3] D. Hostler, S. Everson-Stewart, T. D. Rea et al., "Effect of real-time feedback during cardiopulmonary resuscitation outside hospital: prospective, cluster-randomised trial." BMJ (Clinical research ed.), vol. 342, p. d512, Feb. 2011.

[4] M. P. Chang, Y. Lu, B. Leroux et al., "Association of ventilation with outcomes from out-of-hospital cardiac arrest." Resuscitation, vol. 141, pp. 174-181, Aug. 2019.

[5] E. Aramendi, Y. Lu, M. P. Chang et al., "A novel technique to assess the quality of ventilation during pre-hospital cardiopulmonary resuscitation.” Resuscitation, vol. 132, pp. 41-46, Nov. 2018.

[6] M. Risdal, S. O. Aase, M. Stavland et al., "Impedancebased ventilation detection during cardiopulmonary resuscitation." IEEE Trans Biomed Eng, vol. 54, pp. 2237-2245, Dec. 2007.

[7] X. Jaureguibeitia, U. Irusta, E. Aramendi et al., "Automatic detection of ventilations during mechanical cardiopulmonary resuscitation," IEEE JBHI, 2020.

[8] H. E. Wang, R. H. Schmicker, M. R. Daya et al., "Effect of a strategy of initial laryngeal tube insertion vs endotracheal intubation on 72-hour survival in adults with out-of-hospital cardiac arrest: a randomized clinical trial," JAMA, vol. 320, no. 8, pp. 769-778, 2018.

[9] U. Irusta, J. Ruiz, S. R. de Gauna et al., "A least meansquare filter for the estimation of the cardiopulmonary resuscitation artifact based on the frequency of the compressions." IEEE Trans Biomed Eng, vol. 56, pp. 1052-1062, Apr. 2009.

[10] Y. Xiao and Y. Tadokoro, "Lms-based notch filter for the estimation of sinusoidal signals in noise," Signal Processing, vol. 46, no. 2, pp. 223-231, 1995.

[11] L. Breiman, "Random forests," Machine Learning, vol. 45, no. 1, pp. 5-32, 2001.

Address for correspondence:

Xabier Jaureguibeitia

Plaza Ingeniero Torres Quevedo 1. 48013 - Bilbao, Spain

xabier.jaureguibeitia@ehu.eus 